\title{
ARTIGO ORIGINAL Céu azul: comunicação para inspirar o comportamento econômico e inovar a Embraer
}

\section{BLUE SKY: COMMUNICATION FOR INSPIRING THE ECONOMIC BEHAVIOR AND INNOVATING EMBRAER}

\section{Ricardo Manini}

Jornalista e economista. Mestre em Divulgação Científica (Labjor/IEL/Unicamp). Doutorando em Política Científica e Tecnológica no Instituto de Geociências da Universidade Estadual de Campinas.

E-mail: rmanini@gmail.com

\section{Graça Caldas}

Jornalista, docente e pesquisadora do Programa de Pós-Graduação do Labjor/Unicamp. PósDoutorado em Políticas Científicas pelo Instituto de Geociências da Universidade Estadual de Campinas. Doutora em Ciências da Comunicação pela Escola de Comunicações e Artes da Universidade de São Paulo. Mestre em Comunicação Científica e Tecnológica pela Universidade Metodista de São Paulo. Bolsista produtividade do CNPq.

E-mail: gcaldas@unicamp.br

Recebido em 21 de abril de 2017. Aprovado em 21 de junho de 2017.

\section{Resumo}

A busca pela inovação foi incorporada aos processos produtivos das empresas modernas para garantir maior competitividade no mercado. Mas a inovação não pode ser vista apenas como uma atividade econômica. A comunicação corporativa é igualmente importante no desenvolvimento dos processos inovadores. Este artigo examina o papel e a relação entre a comunicação e a inovação a partir da atuação da empresa Embraer, com o programa Desafio Innova, que incentiva seus funcionários, por meio da comunicação, a elaborarem soluções inovadoras para seus problemas. O referencial teórico deste trabalho se baseia principalmente em pesquisadores como North, Johnson-Laird e Allen. Os resultados desta pesquisa apontam que a experiência da Embraer pode ser útil para outras empresas.

Palavras-chave: Comunicação corporativa. Comunicação para inspiração. Inovação. 


\section{Abstract}

The search for innovation has been incorporated in the production processes of modern enterprises to achieve increased competitiveness in the market. However, innovation cannot be seen only as an economic activity. Corporate communication is also important in developing innovative processes. This article analyzes the role and the relation between communication and innovation in the activity of Embraer with the program Desafio
Innova, which encourages its employees, through communication, to create innovative solutions to its problems. Our theoretical framework is mainly based on researchers as North, Johnson-Laird and Allen. The conclusions of this article show that the program may be useful to other enterprises.

Keywords: Corporate communication. Communication for inspiration. Innovation.

\section{Introdução}

A prática da inovação ganhou importância ímpar nas últimas décadas. A busca pela inovação de produtos e processos tornou-se essencial no mundo corporativo. $\mathrm{O}$ avanço da tecnologia e a globalização da economia determinaram uma nova dinâmica no mercado consumidor, obrigando as empresas a investirem cada vez mais na inovação para manter sua competitividade.

Cada vez mais, governos, universidades e empresas se preocupam em aumentar a atividade inovadora como modo de impulsionar a economia. Em parte, essa maior preocupação com a inovação está ligada a estudos na área econômica que demonstram, desde ao menos a década de 1950 (SOLOW, 1957; SWAN, 1956), que processos inovadores podem aumentar a produtividade, melhorar o desempenho da empresa no mercado e contribuir para o crescimento da economia de um país.

Há mais de 15 anos, em 1999, a revista inglesa The Economist publicou uma reportagem intitulada "Industry gets religion", cujo primeiro parágrafo relaciona a inovação a uma nova religião da indústria. Com essa metáfora, o texto procurou mostrar a crença inabalável nos processos inovadores. Desde então, a preocupação com estes por parte da indústria só aumentou, de modo que o texto continua atual. De acordo com a reportagem,

a inovação se tornou a nova religião industrial do fim do século XX. A indústria a enxerga como chave para aumentar lucros e market share. Governos automaticamente recorrem a ela quando tentam consertar a economia. Por todo o mundo, a retórica da inovação tem substituído a retórica do pós-guerra da economia do bem-estar ("welfare economics"). É 
a nova teologia que une a esquerda e a direita do espectro político, diz Gregory Daines, da Universidade de Cambridge (INDUSTRY..., 1999).

No Brasil, o reconhecimento da inovação no desenvolvimento do país não é diferente. Diversos mecanismos têm sido criados há mais de 20 anos para estimular a inovação nas empresas. Como explicam Corder e Buainain (2013, p. 1), “o Brasil conta atualmente com todos os instrumentos para apoiar, financiar e incentivar a inovação [...]. Essa estrutura foi o resultado da política de Ciência, Tecnologia e Inovação implantada no Brasil a partir de fins dos anos de 1990”.

Na última década, leis importantes - como a Lei da Inovação ( $\left.n^{\circ} 10.973 / 04\right)$ e a Lei do Bem ( $n^{\circ}$ 11.195/05) - foram elaboradas pelo Governo Federal e aprovadas pelo Congresso Nacional para incentivar a inovação. Novos parques tecnológicos foram instalados em diferentes regiões do país. Mais recentemente, em 2013, foi criada a Empresa Brasileira de Pesquisa e Inovação Industrial (Embrapii), que exerce papel relevante no ecossistema nacional de inovação. O Banco Nacional de Desenvolvimento Econômico e Social (BNDES) concedeu, durante os últimos anos, vultosos investimentos para a atividade inovadora. Por fim, mas não menos importante, pesquisas passaram a ser feitas para mensurar os resultados desses esforços, como a Pesquisa de Inovação (Pintec) realizada nos anos de 2000, 2003, 2005, 2008 e 2011.

Entre as empresas com sede no Brasil que se destacam em rankings globais de inovação estão: Empresa Brasileira de Aeronáutica (Embraer), Totvs, Companhia Paulista de Força e Luz (CPFL Energia), Braskem, WEG, Vale, Petrobras, Itautec, entre outras. Para este artigo, com base no produto desenvolvido, de alta complexidade tecnológica, e também devido a resultados alcançados em alguns rankings importantes de inovação mundial, selecionamos a Embraer para um estudo de caso.

A metodologia utilizada, além do processo de escolha da empresa, foi o levantamento e a análise bibliográfica pertinente ao assunto, incluindo trabalhos acadêmicos e relatórios anuais da empresa dirigidos a acionistas, nos quais se especificam quais atividades foram desenvolvidas no período. Duas fontes da Embraer, da área de comunicação e de inovação, também foram entrevistadas.

\section{Comunicação e inovação}

Neste recorte da pesquisa procuramos mostrar que a inovação não é apenas uma atividade econômica, mas também uma atividade que tem a comunicação como fundamento. Esforços de comunicação são fundamentais para que processos inovadores ganhem fôlego e gerem resultados. Mais do que isso, a comunicação pode assumir um papel 
passivo, embora necessário, ou um papel ativo na criação de um novo conhecimento, contribuindo para o processo inovador das corporações.

Assumimos como um dado que a "faísca" para a criação de novo conhecimento ocorre sobretudo no plano individual, ainda que esteja sujeito a influências coletivas. Essa dinâmica ocorre pela manipulação interna de modelos mentais e da quebra de instituições, o que pode ser compreendido como um comportamento econômico inovador, dentro do arcabouço teórico da Nova Economia Institucional (NORTH, 1990). Em momentos anteriores e posteriores a esse comportamento, a comunicação serve como ativo de amplo valor.

O artigo está dividido em cinco sessões, além desta introdução. Na primeira parte, analisamos o que é um comportamento econômico inovador, o que são modelos mentais e o que são instituições. Em seguida, relacionamos, de modo mais aprofundado, o comportamento econômico com processos de comunicação. Na sequência, discutimos os tipos de comunicação científica e como a comunicação para inspiração pode catalisar o comportamento inovador. Finalmente, abordamos o caso de um programa da Embraer, o Desafio Innova, e verificamos a compatibilidade desse programa com o conceito de "comunicação para inspiração", bem como a viabilidade de o programa ser referência para outras empresas que lidam com inovação. Por fim, há uma breve conclusão.

\section{Comportamento econômico, modelos mentais e novos conhecimentos}

A economia é uma ciência social. Ela se preocupa com os modos pelos quais os agentes se organizam para suprir suas necessidades e aumentar seu bem-estar. Todo comportamento econômico é um comportamento humano. A especificidade do comportamento econômico reside em ser direcionado a lograr metas econômicas, por meio de influências e limites relacionados à esfera econômica.

Essa esfera não existe sozinha no espaço e recebe influência da cultura, da sociedade e da política. É caracterizada sobretudo pela produção, distribuição e consumo de bens e serviços. Lida com a administração de recursos escassos e com a atividade inovadora, ou seja, pela atividade responsável por introduzir novos processos, bens e serviços. A introdução exitosa desses elementos no mercado ocorre com sua produção, distribuição e consumo, gerando dividendos para a empresa e o país.

Pode-se argumentar que o comportamento econômico inovador é um comportamento humano caracterizado pela criação e introdução bem-sucedida de uma ideia, sob a forma de bem ou serviço, no mercado ("inovação de produto") ou em linhas produtivas 
("inovação de processos"). A Nova Economia Institucional, por meio do conceito basilar para a área, o de instituições, procura explicar o comportamento inovador.

Mas o que são instituições? Como elas funcionam? A que lógicas obedecem? De acordo com North (1990, p. 3), "as instituições são as regras do jogo em uma sociedade, ou, mais formalmente, são as restrições concebidas pelos indivíduos que moldam a interação humana". Já no dizer de Hodgson (2006, p. 2), "instituições são sistemas de regras sociais estabilizadas e predominantes que estruturam a interação social".

Embora diferentes, as duas concepções sobre o conceito indicam dois aspectos básicos no comportamento das instituições: 1) elas impõem regras sociais e, assim, 2) moldam a dinâmica das relações humanas. Para Hodgson (2006), exemplos de instituições são a linguagem, a moeda, o direito, os sistemas de pesos e medidas, e até mesmo as normas de etiqueta à mesa. Até mesmo um modo de atravessar a rua pode ser chamado de instituição, na medida em que existe, por exemplo, uma tendência socialmente compartilhada dos pedestres de olhar para os dois lados antes de cruzá-la.

Ou seja, as instituições não precisam ser formais, estar escritas em códigos ou serem normas jurídicas. Em realidade, a maioria delas é informal, aceita socialmente e, muitas vezes, nem sequer percebida como instituição, embora condicione boa parte da vida em sociedade. Uma instituição molda também comportamentos econômicos e tem forte relação com comportamentos inovadores.

A origem das instituições, como definidas aqui, está em modelos mentais compartilhados entre indivíduos. A existência desses modelos tem sido imaginada desde meados do século 19, por exemplo em alguns trabalhos de Peirce, filósofo e matemático americano, sobre lógica. Conforme afirma Johnson-Laird (2005, p. 181),

Um princípio da moderna teoria de modelos mentais é que o modelo tem a mesma estrutura da situação que ele representa. Como uma maquete feita por um arquiteto, ou um modelo molecular criado por um biólogo, as partes do modelo e as suas relações estruturais correspondem àquelas do que ele representa [...]. O avô intelectual da teoria é Charles Sanders Peirce.

Craik (1943) deu traços subsequentes à teoria. Ele afirmou, ao se referir a modelos mentais, que os indivíduos pensam ao manipular esses modelos. Johnson-Laird (1980) argumentou, nessa perspectiva, que os seres humanos criam modelos mentais do mundo com base na compreensão de discursos, e essa criação ocorre por meio de processos mentais tácitos.

Desse modo, pode-se dizer que a linguagem é responsável por permitir que os indivíduos criem modelos mentais, com os quais raciocinam. Esses modelos, elaborados 
por processos da comunicação, são compartilhados. O compartilhamento gera regras sociais, ou seja, instituições. Essa lógica é importante para o arcabouço teórico da Nova Economia Institucional.

Sobre o conceito de instituições, Denzau e North (1994, p. 2) argumentam que "modelos mentais são as representações internas que sistemas cognitivos individuais criam para interpretar o ambiente e as instituições são os mecanismos externos (à mente) que os indivíduos criam para estruturar e ordenar o ambiente". Em seguida, afirmam também, em uma passagem fundamental para este trabalho, que

alguns tipos de modelos mentais são compartilhados entre pessoas. Indivíduos diferentes com modelos similares os acionam para melhor se comunicar e compartilhar seus aprendizados. [...] As instituições podem então ser vistas como classes de modelos mentais compartilhados (DENZAU; NORTH, 1994, p. 2, grifo nosso).

É assim que a linguagem e os modelos mentais compartilhados (MMC) contribuem para uma melhor comunicação, a qual permite a transferência de aprendizados. Nesse sentido, pode-se dizer que:

$$
\mathrm{MMC} \rightarrow \text { Comunicação } \rightarrow \text { Compartilhamento de aprendizado } \rightarrow \text { MMC }
$$

Esse processo é, portanto, circular. Mas no caso de ambientes inovadores, surge com frequência um novo conhecimento, que pode levar a um novo produto, a um novo processo ou a melhorar consideravelmente um produto ou processo. Assim, pode-se falar em uma outra dinâmica, que inclua o "novo conhecimento" (NC).

$$
\begin{aligned}
\mathrm{MMC} & \rightarrow \text { Com. } \rightarrow \text { Compartilhamento de aprendizado } \rightarrow \\
& \rightarrow \mathrm{MMC} \rightarrow \mathrm{NC} \rightarrow \text { Com. } \rightarrow \text { MMC }
\end{aligned}
$$

É importante perceber que esse novo conhecimento, conforme visto pela Nova Economia Institucional, não surge diretamente de um grupo. É razoável imaginar que, por mais que um grupo influencie o indivíduo por meio de ideias, novos conhecimentos são gerados no plano individual, e não de modo coletivo. A esse respeito, tornam-se de extrema importância as ideias expressas por Nonaka (1991). De acordo com os autores japoneses,

novo conhecimento só se inicia com o indivíduo. Um pesquisador brilhante tem um insight que leva a uma nova patente. A intuição sobre tendências de mercado de um gerente de nível 
médio se torna catalisadora para a concepção de um novo produto. Um trabalhador de chão de fábrica se baseia em anos de experiência para produzir uma nova inovação de processo. Em cada caso, o conhecimento individual de uma pessoa se transforma em conhecimento organizacional valioso para a organização como um todo (NONAKA, 1991, p. 97).

Isso significa dizer que

$$
\mathrm{NC} \text { (indivíduo) } \rightarrow \text { Comunicação } \rightarrow \mathrm{MMC} \text { (coletivo) }
$$

Ou, para termos a visão de todo o processo, o esquema pode ser traçado assim:

$$
\begin{aligned}
\mathrm{MMC} & \rightarrow \text { Com. Compartilhamento de Aprendizado } \rightarrow \\
& \rightarrow \mathrm{MMC} \rightarrow \mathrm{NC}(\mathrm{i}) \rightarrow \text { Com. } \rightarrow \mathrm{MMC}
\end{aligned}
$$

O elemento “(i)", ao lado de "NC", ressalta a importância do indivíduo em relação ao novo conhecimento. É esse indivíduo que descobre e é o responsável por comunicá-lo ao grupo em um primeiro momento. Após a primeira comunicação, que é de uma pessoa para um grupo, a comunicação do novo conhecimento tende a contar com mais emissores, deixando de ser responsabilidade exclusiva do descobridor pioneiro, podendo, então, ser coletivo.

Isso ocorre porque, para colocar em uso um novo conhecimento, muitas vezes a equipe reparte responsabilidades de acordo com as funções de cada integrante, já estabelecidas na hierarquia e na organização corporativa. A comunicação deixa de ser, nesse caso, de indivíduo para o coletivo e passa a ser de coletivo para coletivo (do grupo para o grupo), em um processo fundamental.

Pode-se esboçar, portanto, uma forte imbricação entre modelos mentais compartilhados (coletivos por excelência), comunicação (em um primeiro momento individual $\rightarrow$ grupo, e depois, grupo $\rightarrow$ grupo) e novo conhecimento (sempre individual). Dessa imbricação surgem inovações, fundamentais para o crescimento de uma empresa. O processo de comunicação, portanto, tem um papel essencial para gerar inovações. Mas antes de abordar esse papel é preciso perquirir como surge o conhecimento individual, isto é, o que representa um comportamento econômico inovador.

\section{O papel da comunicação}

Um indivíduo de comportamento exclusivamente moldado por instituições tem, por definição, um comportamento-padrão, inserido nas "regras do jogo" social. Por essa 
perspectiva, ao seguir todas as regras, formais e informais, o espaço para o exercício da criatividade tende a se reduzir ou até mesmo a se esgotar.

De acordo com vários autores, a criatividade envolve a geração de novos comportamentos aos quais subjaz certa utilidade ou qualidade (GUILFORD, 1968; MALTZMAN, 1960; SHALLEY, 1991). Se o indivíduo segue todas as instituições, sem transgredi-las, ele segue as normas institucionais e não apresenta novos comportamentos criativos capazes de gerar processos inovadores, que de certa forma implicam em rupturas.

Em relação especificamente ao comportamento inovador, de acordo com Dequech (2003, p. 157),

o comportamento inovador é ao menos em parte não convencional (i.e, não institucional). Ainda assim, é difícil pensar em uma pessoa que age de modo completamente não convencional. Existem tantas convenções, e é muito difícil romper com todas ao mesmo tempo. O empreendedor ou firma schumpeteriano(a) irá continuar a seguir algumas convenções ao romper com outras. Uma inovação implica em quebrar com modos convencionais de fazer alguma coisa, mas isso não necessariamente viola muitas outras convenções que existem em relação a uma firma, como, por exemplo, direitos, deveres, hierarquia, práticas contábeis, e assim por diante.

Pode-se, portanto, afirmar que uma inovação resulta de um comportamento desviante em relação às instituições. Por desviante, indica-se um comportamento que viola alguma instituição de um tempo e espaço definido. No caso do comportamento inovador, o desvio resulta em uma inovação, na criação de um novo processo, bem ou serviço, ou, ao menos, em uma melhora substantiva do processo, bem ou serviço já existente.

São diversos os motivos que levam a esse comportamento, e eles variam da vontade de exercitar a criatividade à possibilidade antevista e imaginada, com certo otimismo, de lucrar com uma inovação, ou seja, com um novo conhecimento aplicável. Na medida em que os indivíduos raciocinam ao manipular modelos mentais internamente, o que ocorre quando um indivíduo rompe com uma instituição (mecanismo externo que ordena o ambiente) é que ele manipula algum modelo mental no plano interno. Assim, muitas vezes, descobre propriedades de objetos e processos por meio dessa manipulação. Como é uma dinâmica individual, ele em geral precisa, conforme vimos, comunicar a descoberta para os demais.

Esse esforço é puramente comunicacional e, exceto no caso em que o agente cria, produz e vende a própria inovação, sem intermediários, a comunicação é essencial. Dado que esse caso, o do empreendedor que trabalha sozinho, sem ajuda interna ou externa, não é de modo algum o mais frequente. O processo de inovação exige, em geral, comunicação interna, ou ao menos com colaboradores. 
Nesse processo, a comunicação é um processo condutor em relação à dinâmica inovadora. Isto é, ela ajuda no processo de inovação, porque permite ao inovador pioneiro transferir um novo conhecimento para demais integrantes de um projeto, mas não é o vetor a provocar a manipulação inicial de um dado modelo mental.

Entretanto, a comunicação pode assumir um papel de maior destaque dentro de uma organização. O processo comunicacional pode, também, ser o vetor responsável pela manipulação do modelo mental de um agente, assumindo assim um caráter mais ativo, por meio da comunicação para inspiração, conceito de Thomas J. Allen (1977), professor de estudos organizacionais do Massachusetts Institute of Technology (MIT/EUA).

\section{Os três tipos de comunicação e a comunicação para inspiração}

De acordo com Allen (1977), a inovação depende da comunicação científica e, considerando apenas essa área de estudo, pode ser dividida em três tipos. O primeiro é a comunicação para coordenação, que existe em praticamente todas as organizações e ajuda a manter os integrantes de uma equipe informados. Ela pode ser resumida no ditado “a mão direita precisa saber o que a mão esquerda está fazendo", isto é, precisa existir comunicação entre as partes para o resultado de um todo.

O segundo tipo é a comunicação para informação, que deve ocorrer para a empresa se manter atualizada a respeito do que ocorre em sua área de expertise. Ou seja, a fim de obter êxito em um ambiente em constante mudança, os integrantes de uma organização precisam se atualizar de modo constante, logo realizam a comunicação para informação.

Por fim, e mais importante para este trabalho, existe a comunicação para inspiração, considerada crítica no processo de inovação em uma corporação. Como Allen e Henn (2007) definem,

o terceiro tipo é a comunicação para inspiração. De modo diferente que a comunicação para informação, que serve ao papel de transferir e transformar conhecimento existente, a comunicação para inspiração é ativa em criar conhecimento. Em uma organização que confia em soluções criativas para problemas, a comunicação para inspiração é absolutamente crítica. É geralmente espontânea e geralmente ocorre entre pessoas que trabalham em unidades organizacionais diferentes, em diferentes projetos, e se apoia em diferentes disciplinas. Esses comunicadores são pessoas que, sob circunstâncias normais, teriam pouco a ver um com o outro e talvez nem sequer interagissem. É uma comunicação transdisciplinar, transfuncional, que permite o desenvolvimento de combinações não usuais de ideias que levam à imaginação e à criatividade (, p. 28, grifo nosso). 
A comunicação para inspiração é, portanto, um processo útil na criação de conhecimento. Ela não apenas comunica uma ideia criativa, mas ajuda na sua elaboração. Assume, assim, um papel ativo. A referência, no excerto, a combinações não usuais de ideias indica que a criação de conhecimento não se dá por meio de um comportamento-padrão, convencional e perfeitamente compatível com instituições.

Embora não exista muita literatura sobre a relação entre esse tipo de comunicação e modelos mentais, pode-se inferir, pelo até aqui exposto, que essa comunicação permite a indivíduos manipularem modelos mentais previamente armazenados. Como consequência, induz a um comportamento inovador, desviante em relação a instituições de uma dada organização, e contribui para soluções criativas de problemas.

Um ponto adicional da comunicação para inspiração é que ela permite à organização, usando recursos humanos à disposição, sem contratar pessoas de fora, encontrar soluções para alguns problemas. Isso porque, se bem conduzidos, os processos de "comunicação para inspiração" permitem que problemas em uma determinada área sejam resolvidos até mesmo por pessoas de áreas diferentes dentro da mesma empresa.

Allen e Henn (2007, p. 11) comentam que "a melhor fonte de ideias técnicas para engenheiros que desenvolvem novos produtos é um colega na mesma organização". No entanto,

essa rica fonte de ideias é significativamente subutilizada. Por quê? Existem muitas razões, mas talvez principalmente por uma simples falta de consciência. Em grandes organizações, a equipe frequentemente está desavisada sobre a diversidade de talentos entre os seus trabalhadores. Nós escutamos muitas histórias de muitas pessoas diferentes sobre como eles procuraram muito e longe por alguém com um tipo particular de conhecimento apenas para descobrir que a pessoa correta está dentro de suas próprias organizações, às vezes no prédio adjacente (Ibid., p. 12).

Nesse sentido, a comunicação para inspiração é necessária para descobrir se em uma organização existe alguém capaz de resolver problemas com os quais a empresa frequentemente se depara. Como esse tipo de comunicação é responsável por criar conhecimento, se há alguém dentro da empresa capaz de criar novos conhecimentos que solucionem problemas, é possível que essa pessoa seja a adequada para um determinado cargo. Argumenta-se então que descobrir e mapear talentos de integrantes de uma organização é fundamental para que ela ganhe tempo, conheça melhor seus recursos humanos e seja melhor gerida.

Com base na discussão teórica desenvolvida até agora, é possível debater o programa interno da Embraer denominado Desafio Innova. Acreditamos, com base na 
literatura examinada e na pesquisa realizada na Embraer, que esse programa seja um referencial importante para empresas que desejam implementar programas de comunicação para inspiração.

A título de contextualização, delineamos brevemente a importância dada pela Embraer a processos inovadores; em seguida, apresentamos os resultados alcançados pela empresa quanto à inovação; na sequência, explica-se o programa Desafio Innova. Com base nos relatórios públicos dirigidos a acionistas, apontamos os principais resultados do programa. Por fim, discute-se a compatibilidade desse programa com o conceito de comunicação para inspiração, observando se há viabilidade de programas semelhantes serem implantados em outras empresas.

\section{A Embraer e o Desafio Innova}

A Embraer foi fundada em 1969 como uma sociedade de economia mista vinculada ao Ministério da Aeronáutica. A prévia existência do Instituto Tecnológico da Aeronáutica (ITA) e do Centro Técnico Aeroespacial (CTA) em São José dos Campos (SP) em muito contribuiu para que a empresa se instalasse no município.

A crise econômica brasileira da década de 1980 trouxe problemas sérios para a empresa, que foi privatizada em meados do decênio seguinte. Na atualidade, a Embraer é uma das mais importantes produtoras de aeronaves do mundo, tendo como concorrentes a Boeing, a Airbus e a Bombardier. Fabrica aviões comerciais, executivos, agrícolas e militares.

A empresa emprega cerca de 19 mil funcionários e tem o capital aberto na Bolsa de Valores brasileira e também na de Nova York. Seu valor de mercado era de R \$20,4 bilhões em março de 2013. Além da unidade de São José dos Campos e de outras no Brasil, a empresa também tem filiais nos Estados Unidos, França, China, Cingapura e Portugal, além de ter como clientes companhias aéreas africanas, asiáticas, europeias e americanas.

A Embraer é frequentemente eleita como uma das companhias com sede no Brasil mais inovadoras. A empresa aparece no ranking de 2012 Global Innovation 1000, da consultoria Booz \& Company (atual Strategy\&), na $859^{\mathrm{a}}$ posição, atrás apenas da Vale, Petrobras, Totvs, CPFL Energia e Gerdau. No ranking de 2014 já melhora substancialmente sua posição, passando para a $492^{\mathrm{a}}$ posição, em relação às brasileiras, atrás apenas da Petrobras e da Vale.

É uma das dez empresas brasileiras mais inovadoras eleitas pela revista americana Forbes (2012) e em ranking semelhante feito pela revista Fast Company (2011), também americana. Em um suplemento de quatro páginas sobre inovação e pesquisa no Brasil, 
a publicação de 16 de abril de 2012 do jornal britânico Financial Times, um dos mais importantes veículos internacionais de negócios, também elenca a Embraer como um caso emblemático de êxito em inovação. Em 2012, a empresa recebeu o prêmio Finep de Inovação, talvez o mais importante prêmio concedido a empresas inovadoras pelo governo brasileiro.

\section{Estratégias inovadoras}

O impulso a processos inovadores é uma estratégia fundamental da empresa. Isso pode ser notado por meio de dados da própria instituição. Em relação a dados qualitativos, a inovação é um dos seis valores da empresa. No site da Embraer, na seção "Filosofia Empresarial", entre os valores listados está "Ousadia e inovação são a nossa marca" (FILOSOFIA..., 2012). Já na parte referente ao "Plano Estratégico", podemos ler o seguinte trecho: "prosseguir como uma organização global e comprometida com a satisfação de seus clientes e a valorização de suas pessoas, que cresce de forma sustentável e é referência em inovação e excelência empresarial" (ESTRATÉGIA..., 2012).

A empresa lista ainda, na parte de "Filosofia Empresarial", o programa Innova Embraer. A respeito desse programa, a empresa afirma em seu portal institucional:

Graças a uma cultura interna e a processos fortemente dedicados ao estímulo à inovação, a Embraer é reconhecida como uma Empresa fértil em tecnologias de ponta [...] A principal ferramenta para garantir um ambiente interno fértil e estimular as ações de inovação na Empresa é o programa Innova, que disponibiliza canais e espaços propícios ao desenvolvimento e à consolidação das ideias inovadoras. A Embraer possui ainda uma equipe dedicada exclusivamente ao estudo e à viabilização de novas tecnologias e de novos processos que possam agregar valor à Empresa. Essa equipe desenvolve, em média, 40 a 50 projetos por ano, baseados em análise periódica das necessidades do mercado e do cenário tecnológico mundial (EMBRAER, 2013).

A participação dos funcionários nos processos de inovação é obtida por meio de alguns mecanismos internos, como o Desafio Innova. Esse programa estabelece que, quando surgir um problema na empresa, uma comunicação, geralmente por e-mail, é feita aos funcionários em geral. Essa comunicação é direcionada a praticamente todas as áreas da empresa. Dessa forma, os funcionários podem participar individual ou coletivamente de novas propostas inovadoras que elaborem um plano para resolver o problema detectado.

Estimular a participação é parte importante das estratégias inovadoras da empresa. Caso o plano seja aprovado por um grupo decisório, o funcionário ou o grupo 
formulador do plano ganha um determinado tempo para elaborar, na prática, a solução. Se funcionar, ele(s) pode(m) até mesmo ser remanejado(s) da sua função original e assumir um novo cargo. A cultura empresarial nos processos inovadores favorece a participação de todos.

Desse modo, a comunicação passa a ser um vetor causal da criação de conhecimento. É por ter acesso a essa comunicação que um determinado funcionário passa a raciocinar sobre possíveis soluções. Ou seja, é a partir disso que ele começa a manipular seus modelos mentais a fim de encontrar uma saída para um obstáculo com o qual a empresa se depara. Essa saída será obtida, em geral, por meio de um comportamento de desvio, de ruptura aos modelos de produção normalmente praticados, como já explicado em termos teóricos na parte inicial do texto. $\mathrm{O}$ receptor da mensagem, portanto, tem enorme importância para o processo de comunicação que visa à atividade inovadora.

Em 2012, foram lançados 5 desafios, que geraram 491 ideias. Destas, 13 entraram em fase de prototipação. Já em 2013, o número de novas ideias por parte dos funcionários, a partir de 12 desafios, foi de 539. Destas, 24 entraram em fase de prototipação e implementação final. No ano de 2014, foram lançados 8 desafios, dos quais nasceram 660 ideias e 17 protótipos. Já no relatório de 2015, o último disponível, a empresa explica de modo um pouco diferente esses dados. Relata que 1.097 empregados foram reconhecidos por 394 sugestões de aprimoramento. Esses números exemplificam bem o papel da comunicação, do estímulo e do reconhecimento na participação ativa dos funcionários em processos inovadores.

É possível perceber, portanto, que a empresa conseguiu, com o Desafio Innova, criar diversos protótipos, que entraram em fase de implementação final, e gerar mais de 1.300 ideias por parte dos funcionários. Esses números não são desprezíveis e mostram a importância do programa.

Acreditamos, portanto, que o Desafio Innova pode ser enquadrado como um tipo de comunicação para inspiração. Por um lado, a comunicação dentro desse programa, como informa a Embraer, é estimulada, enquanto para Allen e Henn (2007) a comunicação para inspiração é "geralmente espontânea", conforme vimos anteriormente.

No entanto, os outros requisitos para esse tipo de comunicação também estão presentes. Usar métodos e estratégicas de comunicação permite conectar pessoas que de outra forma talvez nem sequer interagissem. Esse é um modelo transdisciplinar porque possibilita o envolvimento de funcionários de diferentes setores e formações, cria novo conhecimento ao despertar a imaginação e a criatividade. Nota-se que Allen (1977) utiliza o termo "geralmente" ao comentar a espontaneidade desse tipo de comunicação. Assim, acredita-se que o fato de o Desafio Innova ser uma comunicação planejada/estimulada, 
logo não espontânea, não elimina a o enquadramento desse programa como comunicação para inspiração.

Em relação à perspectiva de o programa Desafio Innova servir de referência para outras empresas, é importante considerar que a empresa que o adotar deva ter algumas características semelhantes à Embraer ou então fazer eventuais adaptações em seus procedimentos e processos, de acordo com seus produtos e sua cultura empresarial.

Lembramos que a Embraer é uma empresa de alta complexidade tecnológica e com quase 20 mil funcionários. Em uma empresa desse porte, que lida com tecnologia de diferentes formas, foram cerca de 50 protótipos originados de mais de mil ideias que surgiram entre os cerca de 19 mil funcionários nos anos verificados (2012-2015).

Assim, acreditamos, com base nos estudos realizados, que esse tipo de estímulo e comunicação dê mais resultados em uma empresa de maior porte, que lide com tecnologia no seu dia a dia, para ter recursos humanos preparados para pensar sobre o corpo de conhecimento tecnológico. Entretanto, o "modelo" de comunicação por inspiração espontâneo ou estimulado pode e deve ser usado em empresas de diferentes portes, com desafios próprios a perseguir.

\section{Considerações finais}

A comunicação é hoje um processo imprescindível para desenvolver novas ideias e processos de inovação nas empresas contemporâneas em meio a uma grande competitividade. Utilizar modelos e estratégias de comunicação interna como um processo condutor de comportamento corporativo pode representar o diferencial para a gestão de uma empresa inovadora e competitiva, com novos produtos tecnológicos, novos processos e novas ideias. Este comportamento inovador, utilizando a comunicação como ativo indutor do processo inovador, também agrega valor empresarial.

O tipo de comunicação para inspiração permite o surgimento de novas ideias ao fazer com que os agentes manipulem seus modelos mentais internos e desviem de comportamentos- padrão pouco criativos, institucionalizados e imobilizadores de novas práticas produtivas nas empresas. Os conceitos de "modelos mentais" e "instituições" são bastante importantes para estudar a inovação no ramo teórico da Nova Economia Institucional.

Pode-se, portanto, concluir, com base no referencial teórico e prático de cultura inovadora da Embraer, que a empresa utiliza de forma eficaz esse tipo de comunicação dentro do programa chamado Desafio Innova. Considerando os anos de 2012 e 2013, esse programa ajudou a gerar mais de mil novas ideias. É resultado direto do 
estímulo à participação dos funcionários para solucionar problemas enfrentados no cotidiano da empresa.

Esse modelo estratégico de comunicação por inspiração da Embraer pode servir como referência para outras empresas. Caso a empresa seja de grande porte e tenha funcionários preparados para lidar com tecnologia, a chance de êxito ao gerar ideias inovadoras aumenta consideravelmente, uma vez que o desafio é proposto e trabalhado, simultaneamente, por funcionários que participam cotidianamente da vida institucional e sentem-se, assim, parte importante do capital empresarial. Pensar em inovação e comunicação de forma simultânea tem se revelado um bom negócio para o mundo corporativo.

\section{Referências}

ALLEN, T. J. Managing the flow of technology: technology transfer and the dissemination of technological information within the R and D organization. Cambridge, MA: MIT Press, 1977.

ALLEN, T.; HENN, G. The organization and architecture of innovation: managing the flow of technology. Abingdon: Routledge, 2007.

CORDER, S.; BUAINAIN, A. M. O financiamento à inovação no Brasil. ComCiência, Campinas, n. 150, 2013.

CRAIK, K. The nature of explanation. Cambridge, MA: Cambridge University Press, 1943.

DENZAU, A. T.; NORTH, D. C. Shared mental models: ideologies and institutions. Kyklos, Hoboken, v. 47, n. 1, p. 3-31, 1994.

DEQUECH, D. Conventional and unconventional behavior under uncertainty. Journal of Post Keynesian Economics, Abingdon, v. 26, n. 1, p. 145-168, 2003.

EMBRAER. Ousadia e inovação são a nossa marca. In: . Relatório anual 2013. São José dos Campos: Embraer, 2013. p. 50-55.

ESTRATÉGIA do negócio. Embraer, São José dos Campos, 2012. Disponível em: < goo.gl/tpf7jv>. Acesso em: 24 out. 2017.

FILOSOFIA empresarial. Embraer, São José dos Campos, 2012. Disponível em: <goo.gl/o8eZa4>. Acesso em: 24 out. 2017.

GUILFORD, J. P. Intelligence, creativity, and their educational implications. San Diego: RR Knapp, 1968.

HODGSON, G. What are institutions? Journal of Economic Issues, v. 40, n. 1, p. 1-25, mar. 2006.

INDUSTRY gets religion. The Economist, London, feb. 1999. Disponível em: <goo.gl/udKALD>. Acesso em: 15 out. 2017.

JOHNSON-LAIRD, P. N. Mental models in cognitive science. Cognitive science, Austin, v. 4, n. 1, p. 71 $115,1980$.

Mental models: towards a cognitive science of language, inference, and consciousness. Cambridge, MA: Harvard University Press, 1983. 
. Mental models and thought. In: HOLYOAK, K. J.; MORRISON, R. G. (Eds.) The Cambridge handbook of thinking and reasoning. Cambridge, MA: Cambridge University Press, 2005. p. 185-208.

MALTZMAN, I. On the training of originality. Psychological Review, Washington DC, v. 67, n. 4, p. 229$242,1960$.

NONAKA, I. The knowledge-creating company. Harvard business review, Boston, v. 69, n. 6, p. 96-104, 1991.

NONAKA, I.; TAKEUCHI, H. The knowledge-creating company: how Japanese companies create the dynamics of innovation. New York: Oxford University Press, 1995.

NORTH, D. C. Institutions, institutional change and economic performance. Cambridge, MA: Cambridge University Press, 1990.

SHALLEY, C. E. Effects of productivity goals, creativity goals, and personal discretion on individual creativity. Journal of Applied Psychology, Washington, DC, v. 76, n. 2, p. 179-185, 1991.

SOLOW, R. Technical change and the aggregate production function. The review of Economics and Statistics, Cambridge, MA, v. 39, n. 3, p. 312-320, 1957.

SWAN, T. W. Economic growth and capital accumulation. Economic record, Hoboken, v. 32, n. 2, p. 334$361,1956$. 